\title{
Detección de anticuerpos séricos de influenza aviar tipo $A$, enfermedad de Newcastle, bronquitis infecciosa y laringotraqueitis infecciosa en aves acuáticas silvestres de tres lagunas andinas del Ecuador
}

\author{
Detection of serum antibodies of avian influenza type A, Newcastle \\ disease, avian bronchitis and laryngotracheitis in wild \\ aquatic birds of three Andean lagoons of Ecuador
}

Nivia Luzuriaga ${ }^{1,3}$, Xiomara Rivera $^{1}$, Richard Zalazar ${ }^{1}$, Nathaly Reyes $^{1}$, Iván Santiana ${ }^{2}$

\section{Resumen}

\begin{abstract}
El objetivo del estudio fue determinar la presencia de anticuerpos séricos frente a cuatro patógenos respiratorios (influenza aviar [AI], enfermedad de Newcastle [NDV], bronquitis infecciosa aviar [IBV] y laringotraqueítis infecciosa aviar [ILTV]) que podrían afectar a las aves acuáticas migratorias y residentes de tres lagunas altoandinas del Ecuador. Se colectaron 153 muestras de sangre de aves de siete especies en las lagunas andinas de Colta, Yambo y Yahuarcocha. La presencia de anticuerpos (Ac) contra IBV e ILTV se detectó por un ELISA indirecto (ELISAi) y por la prueba de inhibición de la hemoaglutinación (HI); para NDV e influenza aviar (H5N1 y H7N3) se usó un ELISAc e HI. La seropositividad a NDV fue de 3.2\% (5/153), habiendo tres casos en Yahuarcocha en el cormorán neotropical (Phalacrocorax brasilianus), un caso en Colta en la focha andina (Fulica ardesiaca) y uno en Yambo en el pato rojizo andino (Oxyura ferruginea). La seropositividad contra AI fue de 13\% (20/153), mayormente en el pato rojizo andino y el zambullidor plateado (Podicceps occipitalis) en Colta, y en el ánade piquiamarillo (Anas georgica), focha andina y pato rojizo andino en Yambo. Asimismo, se encontró Ac séricos contra IBV en dos cormoranes en Yahuarcocha. No se encontraron anticuerpos contra ILTV.
\end{abstract}

Palabras clave: anticuerpos; aves acuáticas; bronquitis aviar; influenza aviar; Newcastle; Lagos Andinos

\footnotetext{
${ }^{1}$ Unidad de Estudios de la Vida Silvestre, Facultad de Medicina Veterinaria y Zootecnia, Universidad Central del Ecuador, Ecuador

${ }^{2}$ Agencia de Regulación y Control Fito y Zoosanitario del Ecuador (AGROCALIDAD), Tumbaco, Quito, Ecuador

${ }^{3}$ E-mail: nluzuriaga@uce.edu.ec
}

Financiamiento: Programa de Proyectos Avanzados- Universidad Central del Ecuador-Proyecto $N^{\circ} 42$ 
The aim of this study was to determine the presence of serum antibodies against four respiratory pathogens (avian influenza [AI], Newcastle disease [NDV], avian infectious bronchitis [IBV] and avian infectious laryngotracheitis [ILT]) that could affect migratory aquatic and resident birds of three high Andean lagoons of Ecuador. Blood samples $(n=153)$ were collected from birds of seven species in the Andean lagoons of Colta, Yambo and Yahuarcocha. The presence of antibodies (Ab) against IBV and ILT was detected by an indirect ELISA (ELISAi) and by the haemagglutination inhibition test (HI); and NDV and avian influenza (H5N1 and H7N3) were detected by an ELISAc and HI. Seropositivity to NDV was 3.2\% (5/153), with three cases in Yahuarcocha in the Neotropical cormorant (Phalacrocorax brasilianus), one case in Colta in the Andean coot (Fulica ardesiaca) and one in Yambo in the Andean reddish duck (Oxyura ferruginea). The seropositivity against AI was 13\% (20/153), mainly in the Andean reddish duck and the Southern silvery grebe (Podicceps occipitalis) in Colta, and in the yellow-billed pintail (Anas georgica), Andean coot and Andean reddish duck in Yambo. Likewise, serum Ab was found against IBV in two cormorants in Yahuarcocha. No antibodies against ILT were found.

Key words: antibodies; waterbirds; avian bronchitis; avian influenza virus; Newcastle disease; Andean Lagoons

\section{INTRODUCCIÓN}

Las aves silvestres son consideradas reservorios o dispersores de patógenos bacterianos o virales, tales como el Paramixovirus de tipo 1 (Enfermedad de NewcastleNDV) e Influenza Aviar (AI) de tipo A, que pueden provocar elevadas tasas de mortalidad y morbilidad en aves domésticas. Generalmente, las aves silvestres y de traspatio adultas suelen ser portadoras asintomáticas de NDV de baja patogenicidad (Elmberg et al., 2017); sin embargo, un eventual brote puede afectar negativamente la estructura de las poblaciones, ya que incrementaría la mortalidad en los individuos jóvenes reduciendo la probabilidad de recambio en la población. Por ejemplo, en la epizootia de 1999 de los Cormoranes de Doble Cresta (Phalacrocorax auritus) en Estados Unidos se estimó una mortalidad de 5000 individuos jóvenes (Glaser et al., 1999; Allison et al., 2005; Diel et al., 2012a).
El contagio de los agentes virales entre aves silvestres y domésticas puede estar facilitado por factores de orden ecológico (cambio climático, alteración del hábitat); inmunológico o biológico (susceptibilidad de las especies, adaptación de los patógenos, contacto con aves infectadas) (Bender et al., 2006; Nallar et al., 2016a). Las evidencias científicas muestran que el hábitat y la susceptibilidad de la especie juegan un rol muy importante; por ejemplo, NDV (Genotipo V) ha sido aislado en aves silvestres (gaviotas, cormoranes y palomas) y aves domésticas (pollos y pavos) de un mismo sector (Diel et al., 2012a), y, en forma similar, mediante técnicas moleculares se ha determinado que los patos migratorios transportan el virus de AI y contagian a las aves domésticas cercanas a los sitios de migración (Jeong et al., 2014).

A nivel de grupos y especies, las aves del orden de los Pelicaniformes (garzas, pelícanos e ibis) y de la familia Scolopacidae (Cormoranes y Vuelvepiedras) son sensibles 


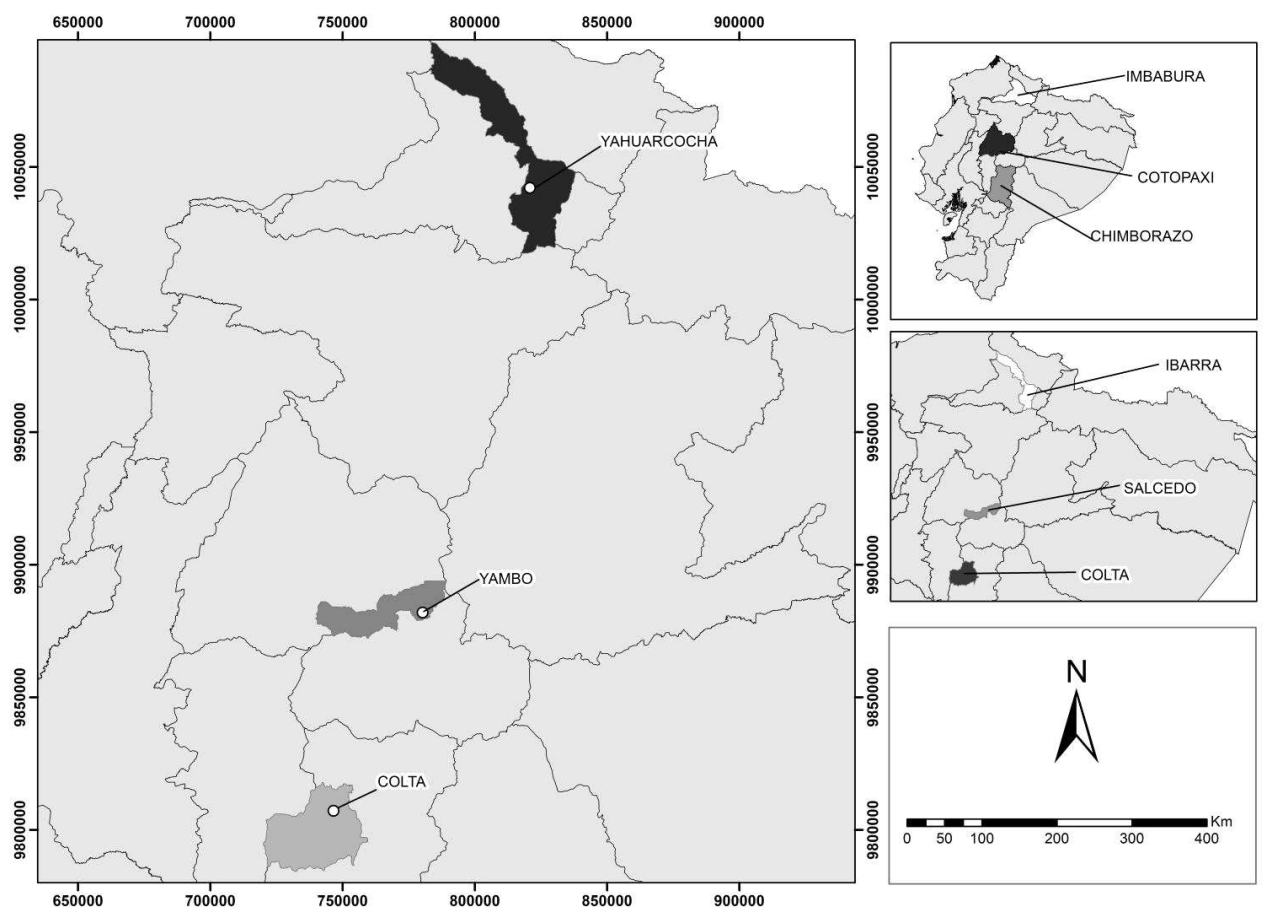

Figura 1. Localización geográfica de la zona de estudio. Presencia de anticuerpos séricos en aves acuáticas silvestres. 2017-2018

a la infección por Coronavirus (CoVs), dentro de la cual se encuentra la Bronquitis Infecciosa Aviar (IBV) (Jordan et al., 2015), así como también las aves comerciales y de traspatio (Villarreal et al., 2010). Adicionalmente, especies del orden de las Anseriformes (patos y gansos) y Caradriformes (gaviotas, playeros y otros) han sido identificados como potenciales reservorios y diseminadores del virus de Influenza A (Brown et al., 2010) debido al alto riesgo de dispersión por los movimientos migratorios característicos de estas especies. Por ejemplo, se ha determinado que el ánade real (Anas platyrhynchos) que migra desde los sitios de reproducción de Norte América hacia las regiones tropicales puede alcanzar tasas de prevalencia superiores al $10 \%$ en los sitios de migración (Hanson et al., 2005; González-Reiche et al., 2012), incrementado así el riesgo de contagio hacia las aves residentes domésticas o de producción masiva.
No se dispone de aproximaciones que permitan identificar la posible circulación de virus asociados a las aves acuáticas de los lagos y lagunas andinas y dada la gran diversidad de aves residentes y migratorias (Guevara et al., 2012) es importante realizar estudios dirigidos que permitan diagnosticar patógenos virales de interés para la salud pública y animal. El objetivo de este estudio fue determinar la presencia de anticuerpos séricos frente a cuatro patógenos respiratorios que podrían afectar a las aves acuáticas migratorias y residentes de tres lagunas altoandinas del Ecuador.

\section{Materiales y Métodos}

\section{Sitios de Estudio}

La laguna Yahuarcocha se encuentra ubicada en la provincia de Imbabura, cantón- 
Ibarra, a 2194 msnm, en la Latitud: -0.37644 y Longitud: -78.11068. La Laguna de Yambo se encuentra ubicada en la provincia de Cotopaxi, cantón Salcedo, a $2681 \mathrm{msnm}$, en la Latitud: -1.09678 y Longitud: $-\mathbf{7 8 . 5 8 7 9 0 . ~}$ La Laguna de Colta se encuentra ubicada en la provincia de Chimborazo, cantón Colta, a 3320 msnm, en la Latitud: -1.72513 y Longitud: - 78.76014 (Figura 1).

\section{Colección de Muestras}

Entre octubre-diciembre de 2017 y enero-marzo de 2018 se colectaron 153 muestras de siete especies de aves acuáticas. Las aves no presentaban sintomatología clínica compatible con las enfermedades motivo de la investigación (Cuadro 1). Todas las capturas se realizaron en sitios de dormideros, durante la noche, utilizando trampa de red y distracción luminosa. Se tomó una muestra de sangre con una jeringa de insulina de la vena braquial o alar $(1 \mathrm{ml})$ y se hizo un hisopado cloacal que fue almacenado y conservado en congelación en medio de transporte estéril de tampón fosfato salino o buffer fosfato salino (PBS). La muestra sanguínea fue colocada en tubos estériles de $10 \mathrm{ml}$, sin anticoagulante. El suero fue colectado por centrifugación luego de 8 a 12 h de coagulación y se almacenó a $-20^{\circ} \mathrm{C}$ para posteriores análisis serológicos, acorde a lo recomendado por González-Acuña et al. (2012).

\section{Análisis de Laboratorio}

Todos los procedimientos han sido desarrollados acorde a las normas técnicas del Manual Terrestre de la Organización Mundial de Sanidad Animal para el Diagnóstico de Enfermedades (OIE, 2013).

\section{Influenza Aviar Tipo A}

La presencia de anticuerpos específicos contra AI se determinó mediante la prueba ELISA competitivo utilizando el estuche comercial Influenza Virus Antibody Test Kit (IDvet Laboratories, Westbrook, USA) siguiendo las recomendaciones del fabricante.
Las muestras que resultaron positivas a esta prueba fueron analizadas a través de la prueba de inhibición de hemaglutinación utilizando como control las cepas inactivadas H5N1 y H7N3 a una dilución de 1: 256, suspendidas en $1 \mathrm{ml}$ de agua destilada y a $4{ }^{\circ} \mathrm{C}$ (National Veterinary Services Laboratories, New York, USA) (Conrad et al., 2018).

\section{Enfermedad de Newcastle}

Los anticuerpos contra el NDV fueron analizados a través de la prueba de inhibición de hemaglutinación, para la cual se usó como control el antígeno NDV a una dilución 1:128 suspendido en agua destilada a $-20{ }^{\circ} \mathrm{C}$ (Laboratorio de Sanidad Animal [AGROCALIDAD], Quito, Ecuador) (Conrad et al., 2018).

\section{Bronquitis Aviar Infecciosa}

La presencia de anticuerpos específicos contra IBV se determinó mediante la prueba ELISA indirecto utilizando el estuche comercial ID Screen ${ }^{\circledR}$ Infectious Bronchitis Virus Indirect (IDvet Laboratories, Westbrook, USA). Los valores fueron considerados positivos con títulos superiores a 690 (Córdoba et al., 2015)

\section{Laringotraqueitis}

Para detectar la presencia de anticuerpos contra laringotraqueítis infecciosa aviar (ILTV) se realizó la prueba de ELISA indirecto, utilizando el estuche comercial ID Screen ${ }^{\circledR}$ ILT Indirect (IDvet Laboratories, Westbrook, USA) (Álvarez et al., 2009).

\section{Inhibición de Hemaglutinación (HI)}

Los antígenos utilizados en esta prueba fueron ajustados para una concentración de 4 UHA (unidades hemaglutinantes) en una solución tamponada (PBS). Los controles de influenza aviar fueron adquiridos en el Laboratorio Nacional de Servicios Veterinarios NVSL -, Ames, EEUU) del USDA-APHIS (United States Department of Agriculture, 
Animal and Plant Health Inspection Service), mientras que el control para ENC fue proporcionado por la Agencia de Regulación y Control Fito y Zoosanitario del Ecuador.

Previo a la prueba, el suero sanguíneo fue tratado con métodos físicos (calor) o químicos (enzimas) con el fin de destruir los inhibidores inespecíficos presentes (GonzálezAcuña et al., 2012). Posteriormente, se dispensaron $25 \mu \mathrm{l}$ de PBS en todos los pocillos de la placa, se adicionaron $25 \mu \mathrm{l}$ de suero sanguíneo en el primer pocillo, y se realizaron diluciones seriadas del mismo a través de los siete pocillos. Además, se añadieron $25 \mu \mathrm{ldel}$ antígeno (NDV o H5N1, H7N3) en todos los pocillos y la placa fue incubada por 30 minutos a temperatura ambiente. Finalmente, se adicionaron $50 \mu \mathrm{l}$ de suspensión de eritrocitos (GR) al $0.5 \%$ en cada pocillo.

La lectura se realizó cuando los glóbulos rojos control sedimentaron en el fondo del pozo, aproximadamente a los 20-30 minutos. La inhibición de hemaglutinación (punto rojo) desde la dilución 1/16 en la placa se consideró positiva para anticuerpos contra NDV e AI como indica el Manual Terrestre de la Organización Mundial de Sanidad Animal para el Diagnóstico de Enfermedades (OIE, 2013; STEAR, 2005).

\section{Resultados y Discusión}

De las 153 muestras analizadas, 20 (14\%) presentaron anticuerpos contra el virus de la Influenza Aviar del tipo A (AI), 2 $(1.4 \%)$ contra el virus de la bronquitis aviar (IBV) y $4(2.8 \%)$ contra el virus de Newcastle (NDV) (Cuadro 1). El mayor número de aves seropositivas a las tres enfermedades se encontraron en Colta, siendo $12 / 20$ de individuos seropositivos a influenza tipo A y $1 / 4$ de individuos seropositivos a NDV. En segundo lugar estuvo Yambo con 6/20 de individuos seropositivos a AI, mientras que Yahuarcocha presentó $2 / 4$ de casos seropositivos para NDV y $2 / 2$ para IBV.
Por especies, el pato rojizo andino (Oxyura ferruginea) presentó el mayor porcentaje de seropositividad a anticuerpos contra $\mathrm{AI}(85 \%, 17 / 20)$, seguido por el zambullidor plateado (Podicceps occipitalis) (10\%, $2 / 20$ ) y por la focha andina (Fulica ardesiaca) $(0.5 \%, 1 / 20)$. Además, se obtuvieron anticuerpos contra NDV en el cormorán neotropical (Phalacrocorax brasilianus) $(50 \%, 2 / 4)$, focha andina $(25 \%, 1 / 4)$ y en el pato rojizo andino (1/4). Adicionalmente, anticuerpos contra IBV fueron detectados en dos cormoranes (2/2) (Cuadros 1 y 2 ).

Los resultados de la prueba de ELISA indirecto (ELISA-i) para IBV determinaron títulos de 1215 y 1318 con una densidad óptica de 0.214 y 0.226 , respectivamente, para los dos cormoranes, siendo considerados como positivos de acuerdo con los valores de referencia (Córdoba et al., 2015).

Adicionalmente, la prueba de inhibición de la hemaglutinación para NDV mostró que dos cormoranes y un pato rojizo andino presentaron títulos de 1:16 y un cormorán y una focha andina presentaron un título de 1:64 (Cuadro 3), siendo en ambos casos considerados positivos de acuerdo con el valor de referencia establecido por la OIE que determina que sueros con una titulación igual o mayor a 1:16 son positivos (OIE, 2004). Ninguna muestra presentó anticuerpos séricos contra laringotraqueitis infecciosa aviar.

Los resultados muestran que el cormorán neotropical (Phalacrocorax brasilianus) es portador de anticuerpos para NDV con $8.33 \%(2 / 24)$ y IBV con $12.5 \%$ (3/24). Por el contrario, ninguna de las otras especies fueron portadoras de anticuerpos contra estos virus.

Los resultados de la investigación muestran la susceptibilidad de cuatro especies de aves comunes de los lagos andinos a infecciones por virus respiratorios. Entre estas especies, las de la familia Anatidae y Podicepedidae presentan anticuerpos contra 
Cuadro 1. Presencia de anticuerpos séricos contra influenza aviar de tipo A (AI), bronquitis aviar infecciosa (IBV) y enfermedad de Newcastle (NDV) en aves acuáticas silvestres de tres lagunas altoandinas del Ecuador

\begin{tabular}{|c|c|c|c|c|c|c|c|c|}
\hline \multirow[t]{2}{*}{$\stackrel{\circ}{:}$} & \multirow[t]{2}{*}{ Aves } & \multirow[t]{2}{*}{$\begin{array}{l}\text { Total } \\
\text { (n) }\end{array}$} & \multicolumn{2}{|c|}{$\begin{array}{l}\text { Influenza } \\
\text { Aviar tipo A } \\
\text { (AI) }\end{array}$} & \multicolumn{2}{|c|}{$\begin{array}{l}\text { Bronquitis } \\
\text { aviar } \\
\text { infecciosa } \\
\text { (IBV) }\end{array}$} & \multicolumn{2}{|c|}{$\begin{array}{l}\text { Enfermedad de } \\
\text { Newcastle } \\
\text { (NDV) }\end{array}$} \\
\hline & & & $\mathrm{n}$ & $\%$ & $\mathrm{n}$ & $\%$ & $\mathrm{n}$ & $\%$ \\
\hline \multirow{4}{*}{$\frac{\pi}{0}$} & Fulica ardesiaca & 10 & - & - & - & - & 1 & 10 \\
\hline & Oxyura ferruginea & 18 & 10 & 56 & - & - & - & - \\
\hline & $\begin{array}{l}\text { Phalacrocorax } \\
\text { brasilianus }\end{array}$ & 1 & - & - & - & - & - & - \\
\hline & Podicceps occipitalis & 10 & 2 & 20 & - & - & - & - \\
\hline \multirow{7}{*}{ 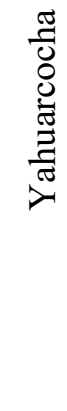 } & Anas georgica & 2 & - & - & - & - & - & - \\
\hline & Ardea cinerea & 5 & - & - & - & - & - & - \\
\hline & Gallinula chloropus & 2 & - & - & - & - & - & - \\
\hline & Fulica ardesiaca & 28 & - & - & - & - & - & - \\
\hline & Oxyura ferruginea & 3 & - & - & - & - & - & - \\
\hline & $\begin{array}{l}\text { Phalacrocorax } \\
\text { brasilianus }\end{array}$ & 28 & - & - & 2 & 14 & 3 & 10 \\
\hline & Podilymbus podiceps & 5 & - & - & - & - & - & - \\
\hline \multirow{3}{*}{ 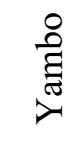 } & Anas georgica & 9 & 6 & 66 & - & - & - & - \\
\hline & Fulica ardesiaca & 14 & 1 & 7.0 & - & - & - & - \\
\hline & Oxyura ferruginea & 4 & 1 & 2.5 & - & - & 1 & 2.5 \\
\hline
\end{tabular}

Cuadro 2. Presencia de anticuerpos séricos contra el virus de influenza aviar tipo A (AI) en aves acuáticas silvestres de dos lagunas altoandinas del Ecuador

\begin{tabular}{llc}
\hline Sitio & Especies & $\begin{array}{c}\text { Influenza aviar } \\
\text { tipo A } \\
(\%)\end{array}$ \\
\hline Laguna de Colta & Pato rojizo andino (Oxyura ferruginea) & $56 \%(10 / 18)$ \\
& Zambullidor plateado (Podicceps occipitalis) & $20 \%(2 / 10)$ \\
Laguna de Yambo & Anade piquiamarillo (Anas georgica) & $66 \%(6 / 9)$ \\
& Focha andina (Fulica ardesiaca) & $7 \%(1 / 14)$ \\
& Pato rojizo andino (Oxyura ferruginea) & $2.5 \%(1 / 4)$ \\
\hline
\end{tabular}


Cuadro 3. Títulos de anticuerpos determinados para la enfermedad de Newcastle (NDV) y bronquitis aviar infecciosa (IBV) en aves acuáticas de tres lagunas altoandinas de Ecuador

\begin{tabular}{lllcc}
\hline \multirow{2}{*}{ Sitio } & \multirow{2}{*}{ Especies } & Código $^{1}$ & IHA & ELISA-i \\
\cline { 3 - 5 } & & (NDV) & (IBV) \\
\hline Yahuarcocha & Phalacrocorax brasilianus & PHABRA-Y-02 & $1 / 16$ & 1215 \\
& & PHABRA-Y-09 & - & 1318 \\
& & PHABRA-Y-13 & $1 / 16$ & - \\
& & PHABRA-Y-55 & $1 / 64$ & - \\
Yambo & Oxyura ferruginea & OXYFER-YB-55 & $1 / 16$ & - \\
Colta & Fulica ardesiaca & FULARD-COL-96 & $1 / 64$ & - \\
\hline
\end{tabular}

IHA: Inhibición de hemaglutinación; ELISA-i: ELISA indirecto; NDV: Newcastle; AIB; Bronquitis infecciosa

${ }^{1}$ PHABRA: Phalacrocorax brasilianus; OXYFER: Oxyura ferruginea; FULARD: Fulica ardesiaca; Y: Laguna de Yahuarcocha; YB: Laguna de Yambo; COL: Laguna de Colta

el virus de AI del tipo A. Estudios previos muestran que algunas de las especies de la Familia Anatidae son reservorios de al menos seis subtipos del virus de AI del mencionado grupo (por ejemplo, Anas disrcors) (González-Reiche et al., 2012), y que factores ecológicos asociados a los sitios de aglomeración (aves migratorias principalmente) pueden favorecer la transmisión por efecto de la densidad (Nallar et al., 2016). La presencia de la cerceta aliazul (Anas discors) en la época de migración en la laguna de Yambo podría explicar en parte los resultados ya que esta especie es sensible a cuatro serotipos de virus de Influenza A (Hanson et al., 2005). Por otro lado, la cercanía a una explotación avícola ( 1 ha) ubicada a menos de $500 \mathrm{~m}$ de la laguna podría ser un factor determinante para la infección de los pollos (Jeong et al., 2014); sin embargo, los estudios de vigilancia activa, tanto en aves industriales como de traspatio, en el Ecuador no han detectado la IA, por lo que se considera exótica en aves domésticas.
En este estudio se observa que el factor migratorio del cormorán neotropical, así como la sensibilidad de las especies del género Phalacrocorax a NDV y IBV podrían representar un riesgo potencial de la transmisión de los virus de dichas enfermedades, pese al bajo porcentaje de positividad encontrado. Los hallazgos de este estudio sugieren la presencia de patógenos virales de importancia zoonótica y clínica en cuatro especies de aves silvestres.

\section{Agradecimientos}

Agradecemos a la Dirección General de Investigación y Postgrado de la Universidad Central por los fondos otorgado al proyecto 42. Al Ministerio del Ambiente por los permisos de investigación. A la Estación de Bomberos de Yahuarcocha por el apoyo logístico. Al laboratorio de Sanidad Animal de la Agencia de Regulación y Control Fito y Zoosanitario por los análisis realizados. Un especial agradecimiento para Washington 
Cárdenas y Augusto Luzuriaga Neira por las revisiones y comentarios al manuscrito. A Margoth Barrionuevo por su apoyo en el laboratorio y al equipo de logística de campo: Leonardo Cedeño, Dana Luzuriaga, Karla Mena y Edwin Reyes.

\section{Literatura Citada}

1. Allison AB, Gottdenker NL, Stallknecht DE. 2005. Wintering of neurotropic velogenic Newcastle disease virus and West Nile virus in double-crested cormorants (Phalacrocorax auritus) from the Florida Keys. Avian Dis 49: 292297. doi: $10.1637 / 7278-091304 \mathrm{R}$

2. Álvarez DC, Usma JA, Jaime J, Vera VJ. 2009. Dinámica serológica del virus de bronquitis Infecciosa en una granja de pollo de engorde del departamento de Cundinamarca. Rev Med Vet Zoo 56: 105-112.

3. Córdoba G, Vera VJ, Correa J, Ramírez GC. 2015. Comportamiento del virus de la bronquitis infecciosa aviar en aves con sintomatología respiratoria provenientes de granjas de producción del departamento de Cundinamarca. Nova 13: 47-64.

4. Bender JB, Hueston W, Osterholm M. 2006. Recent animal disease outbreaks and their impact on human populations. J Agromedicine 11: 5-15. doi: 10.1300/ J096v11n01_02

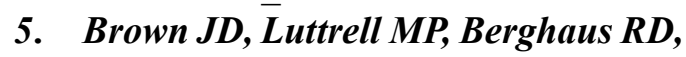
Kistler W, Keeler SP, Howey A, Wilcox $B$, et al. 2010. Prevalence of antibodies to type A influenza virus in wild avian species using two serologic assays. J Wildlife Dis 46: 896-911. doi: 10.7589/ 0090-3558-46.3.896

6. Cifuentes-Rincón A, Lopes PD, San Miguel RA. 2016. Genotyping of news variants of the avian infectious bronchitis virus from Tolima department, Colombia. Rev MVZ Cordoba 21: 5500-5510. doi: $10.21897 / \mathrm{rmvz} .824$
7. Conrad SK, Narwold DR. 2018. Detection of hemagglutinating viruses. VIRPRO. New York: USDA. [Internet]. Available in: https://www.aphis.usda.gov/ animal_health/vet_biologics/publications/ VIRPRO0096.pdf

8. Diel DG, Miller PJ, Wolf PC, Mickley RM, Musante AR, Emanueli DC, Shively KJ, et al. 2012. Characterization of Newcastle disease viruses isolated from cormorant and gull species in the United States in 2010. Avian Dis 56: 128-133. doi: 10.1637/9886-081111-Reg.1

9. Elmberg J, Berg C, Lerner H, Waldenström J, Hessel R. 2017. Potential disease transmission from wild geese and swans to livestock, poultry and humans: a review of the scientific literature from a One Health perspective. Infect Ecol Epidemiol 7: 1300450. doi: 10.1080/20008686.2017.1300450

10. Glaser LC, Barker IK, Weseloh DV, Ludwig J, Windingstad RM, Key DW, Bollinger TK. 1999. The 1992 epizootic of Newcastle disease in double-crested cormorants in North America. J Wildlife Dis 35: 319-330. doi: 10.7589/0090-355835.2.319

11. González-Acuña D, Gaete Á, Moreno L, Ardiles K, Cerda-Leal F, Mathieu C, Ortega R. 2012. Anticuerpos séricos contra la enfermedad de Newcastle e influenza aviar en aves rapaces de Chile. Rev MVZ Cordoba 17: 3118-3124. doi: $10.21897 / \mathrm{rmvz} .210$

12. González-Reiche AS, MoralesBetoulle ME, Álvarez D, Betoulle JL, Müller ML, Sosa SM, Pérez DR. 2012. Influenza A viruses from wild birds in Guatemala belong to the North American Lineage. Plos One 7: e32873. doi: 10.1371/journal.pone.0032873

13. Guevara EA, Santander T, Duivenvoorden JF. 2012. Seasonal patterns in aquatic bird counts at five Andean lakes of Ecuador. Waterbirds 35: 636-641. doi: 10.1675/063.035.0413 
14. Hanson BA, Swayne DE, Senne DA, Lobpries DS, Hurst J, Stallknecht DE. 2005. Avian influenza viruses and paramyxoviruses in wintering and resident ducks in Texas. J Wildlife Dis 41: 624-628. doi: 10.7589/0090-3558-41.3.624

15. Jeong J, Kang HM, Lee EK, Song BM, Kwon YK, Kim HR, Choi KS, et al. 2014. Highly pathogenic avian influenza virus (H5N8) in domestic poultry and its relationship with migratory birds in South Korea during 2014. Vet Microbiol 173: 249-257. doi: 10.1016/ j.vetmic.2014.08.002

16. Jordan BJ, Hilt DA, Poulson R, Stallknecht DE, Jackwood MW. 2015. Identification of Avian Coronavirus in wild aquatic birds of the Central and Eastern USA. J Wildlife Dis 51: 218-221. doi: 10.7589/2014-03-070

17. Nallar R, Papp Z, Leighton FA, Epp T, Pasick J, Berhane Y, Lindsay R, Soos $C$. 2016. Ecological determinants of Avian Influenza Virus, West Nile Virus, and Avian Paramyxovirus infection and antibody status in blue-winged teal
(Anas Discors) in the Canadian prairies. J Wildlife Dis 52: 33-46. doi: 10.7589/ 2013-07-191

18. [OIE] Organización Mundial de Sanidad Animal. 2004. Manual de las pruebas de diagnóstico y de las vacunas para los animales terrestres (mamíferos, aves y abejas) Vol I. [Internet]. Disponible en: http://www.oie.int/doc/ged/ d6508.pdf

19. [OIE] Organización Mundial de Sanidad Animal. 2013. Newcastle disease. Aetiology, epidemology, diagnosis, prevention and control references. [Internet]. Available in: http://www.oie.int/fileadmin/Home/eng/Animal_Health_in_the_World/docs/pdf/Disease_cards/NEWCAST-LE_DISEASE.pdf

20. Villarreal LY, Sandri TL, Souza SP, Richtzenhain LJ, de Wit JJ, Brandao PE. 2010. Molecular epidemiology of avian infectious bronchitis in Brazil from 2007 to 2008 in breeders, broilers, and layers. Avian Dis 54: 894-898. doi: 10.1637/9218-121709-Reg.1 